\title{
Two Characterizations of Hypercubes
}

\author{
Juhani Nieminen, Matti Peltola and Pasi Ruotsalainen \\ Department of Mathematics, University of Oulu \\ University of Oulu, Faculty of Technology, Mathematics Division, \\ P.O. Box 4500, 90014 Oulun yliopisto, Finland \\ ujuhani.nieminen@ ee.oulu.fi; matti.peltola@ee.oulu.fi; \\ pasi.ruotsalainen@ee.oulu.fi
}

Submitted: Jun 11, 2008; Accepted: Apr 20, 2011; Published: Apr 29, 2011 Mathematics Subject Classification: 05C75

\begin{abstract}
Two characterizations of hypercubes are given: 1) A graph is a hypercube if and only if it is antipodal and bipartite (0,2)-graph. 2) A graph is an $n$ hypercube if and only if there are $n$ pairs of prime convexes, the graph is a prime convex intersection graph, and each intersection of $n$ prime convexes (no one of which is from the same pair) is a vertex.
\end{abstract}

\section{Introduction}

Hypercubes constitute a very remarkable class of graphs especially for transmitting communication and therefore each characterization of hypercubes offers a new point of view to use and construct hypercubes.

The class of $(0,2)$-graphs is a subclass of strongly regular graphs studied in the theory of combinatorial design. It was introduced in [6] and intensively studied in [3] and in [4].

We begin with some basic properties of antipodal graphs (see also [7]). Then we prove that a graph is a hypercube if and only if it is an antipodal, bipartite $(0,2)$ graph. This characterization gives another characterization of hypercubes by using prime convex intersection graphs.

The graphs $G=(V, E)$ considered here are finite, connected and undirected without loops and multiple edges. The set $V$ is the set of vertices and $E$ the set 
of edges in $G$. A shortest $u-v$ path is called a $u-v$ geodesic and $d(u, v)$ is its length. The interval $[u, v]$ is the set of all vertices locating on any $u-v$ geodesic. By $N(u)$ we denote the set of neighbours of $u$, i.e $N(u)=\{v \mid d(u, v)=1\}$ and by $\operatorname{deg}(u)$ the cardinality of $N(u)$. The diameter of a graph $G$ is denoted by $\operatorname{diam}(G)=$ $\max \{d(u, v) \mid u, v \in V\}$. A graph $G=(V, E)$ is called antipodal, if for every vertex $u$ there exists - a necessarily unique - vertex $u^{\prime}$ called the antipode of $u$, such that $\left[u, u^{\prime}\right]=V$, see [1] and [5].

\section{Bipartite and antipodal graphs}

We give a sufficient conditions for an antipodal bipartite graph to be a regular one. First we give two basic properties of antipodal graphs (see also [7]).

Lemma 1 An antipodal graph $G=(V, E)$ is bipartite if and only if for any two adjacent vertices $u$ and $v$ of $G$ intervals $\left[u, v^{\prime}\right]$ and $\left[v, u^{\prime}\right]$ constitute a partition of $V$.

Proof. Assume first that $G$ is bipartite. If $u$ and $v$ are adjacent vertices then let $V_{u}=\{w \in V \mid d(u, w)<d(v, w)\}=\{w \in V \mid d(u, w)+1=d(v, w)\}$ and $V_{v}=$ $\{w \in V \mid d(v, w)<d(u, w)\}=\{w \in V \mid d(v, w)+1=d(u, w)\}$. Since $G$ is bipartite, the vertex sets $V_{u}$ and $V_{v}$ constitute a partition of $V$. Moreover, since $\left[v, v^{\prime}\right]=V$, it follows that for every $w \in V_{u}$ we have $w \in\left[u, v^{\prime}\right]$, thus $V_{u}=\left[u, v^{\prime}\right]$. Analogously $V_{v}=\left[v, u^{\prime}\right]$.

Assume that $\left[u, v^{\prime}\right] \cup\left[v, u^{\prime}\right]=V$ and $\left[u, v^{\prime}\right] \cap\left[v, u^{\prime}\right]=\emptyset$ for any two adjacent vertices $u$ and $v$ of $V$. Let $a \in V, V_{1}=\{x \in V \mid d(a, x)$ is odd $\}$ and $V_{2}=\{x \in$ $V \mid d(a, x)$ is even $\}$. Assume first, that there are two adjacent vertices $x, y \in V_{2}$. Let $d(a, x)=2 m$ and $d(a, y)=2 n$. If $m<n$, then, because $x$ and $y$ are adjacent, we have $2 n=d(a, y) \leq d(a, x)+d(x, y)=1+2 m<2 n$; a contradiction. The case $n<m$ is analogous, and accordingly, $n=m$ and $d(a, y)=d(a, x)$. If $a \in\left[x, y^{\prime}\right]$, then $d\left(x, y^{\prime}\right)=d(x, a)+d\left(a, y^{\prime}\right)=d(a, y)+d\left(a, y^{\prime}\right)=\operatorname{diam}(G)$, whence $x^{\prime}=y^{\prime}$, This is a contradiction, since the antipode of a vertex is unique and thus $a \notin\left[x, y^{\prime}\right]$. Similarly we see that $a \notin\left[x^{\prime}, y\right]$, and accordingly, $a \notin\left[x, y^{\prime}\right] \cup\left[y, x^{\prime}\right]$, which is a contradiction. Thus the assumption that there are two adjacent vertices in $V_{2}$ is false and any two vertices $x, y \in V_{2}$ are nonadjacent. Similarly we can prove that any two vertices $x, y$ of $V_{1}$ are nonadjacent. Thus $G$ is bipartite.

Lemma 2 If a graph $G=(V, E)$ is antipodal, then vertices $u$ and $v$ of $G$ are adjacent if and only if $u^{\prime}$ and $v^{\prime}$ are adjacent. Moreover $\operatorname{deg}(u)=\operatorname{deg}\left(u^{\prime}\right)$ for all $u \in V$. 
Proof. Assume that $u$ and $v$ are adjacent vertices. It suffices to prove, that $u^{\prime}$ and $v^{\prime}$ are adjacent. Assume on the contrary, that there exists a vertex $z$ on the $u^{\prime}-v^{\prime}$ geodesic such that $z \neq u^{\prime}, v^{\prime}$. Because $u^{\prime} \in\left[v, v^{\prime}\right]$ and $z \in\left[u^{\prime}, v^{\prime}\right]$, there exists a $v-v^{\prime}$ geodesic through $u^{\prime}$ and $z$, and thus $d\left(v, v^{\prime}\right)=d\left(v, u^{\prime}\right)+d\left(u^{\prime}, z\right)+d\left(z, v^{\prime}\right) \geq d\left(v, u^{\prime}\right)+2$. On the other hand, because $v \in\left[u, u^{\prime}\right]$ and because $v$ is adjacent to $u$, we have $d\left(u, u^{\prime}\right)=1+d\left(v, u^{\prime}\right)$. Because $G$ is antipodal, we have $d\left(v, v^{\prime}\right)=d\left(u, u^{\prime}\right)=\operatorname{diam}(G)$ and thus $d\left(v, v^{\prime}\right)=d\left(u, u^{\prime}\right)=1+d\left(v, u^{\prime}\right) \geq d\left(v, u^{\prime}\right)+2$; a contradiction.

The following theorem gives a sufficient condition for an antipodal bipartite graph to be regular.

Theorem 3 Let $G=(V, E)$ be an antipodal bipartite graph. If for any two adjacent vertices $u$ and $v$ of $G$ there exists a (graph) isomorphism $f$ from the subgraph $G_{1}$ induced by $\left[v, u^{\prime}\right]$ onto the subgraph $G_{2}$ induced by $\left[u, v^{\prime}\right]$ such that $f(x)=y$ for any two adjacent vertices $x \in G_{1}$ and $y \in G_{2}$, then $G$ is regular.

Proof. Let $u$ and $v$ be adjacent vertices. According to the isomorphism $f, f(u)=$ $v$ and $\operatorname{deg}(u)=\operatorname{deg}(v)$. Because $u^{\prime}$ and $v^{\prime}$ also are adjacent, $u^{\prime} \in\left[v, u^{\prime}\right]$ and $v^{\prime} \in$ $\left[u, v^{\prime}\right]$, we have $f\left(u^{\prime}\right)=v^{\prime}$ and $\operatorname{deg}\left(u^{\prime}\right)=\operatorname{deg}\left(v^{\prime}\right)$. This implies, by Lemma 2 , that $\operatorname{deg}\left(u^{\prime}\right)=\operatorname{deg}(u)=\operatorname{deg}(v)=\operatorname{deg}\left(v^{\prime}\right)$, for any two adjacent vertices $u$ and $v$. If $z$ is adjacent to $u$, then by repeating the proof above we have $\operatorname{deg}(z)=\operatorname{deg}(u)$, and further, $\operatorname{deg}(z)=\operatorname{deg}(u)=\operatorname{deg}(v)$. Because $G$ is connected, the result follows.

\section{Spherical graphs}

A graph $G=(V, E)$ is spherical if for any two vertices $u$ and $v$ and for any $z \in[u, v]$ there exists precisely one vertex $z_{1}$ such that $\left[z, z_{1}\right]=[u, v]$. In $[6]$ a graph $G=(V, E)$ is defined as a $(0, \lambda)$-graph if any two distinct vertices $u$ and $v$ have exactly $\lambda$ common neighbours or none at all. Clearly in a $(0,2)$-graph for any two vertices $u$ and $v$ such that $d(u, v)=2$ and for any $z \in[u, v]$ there exists precisely one vertex $z_{1}$ such that $\left[z, z_{1}\right]=[u, v]$.

Theorem 4 A graph $G=(V, E)$ is a hypercube if and only if $G$ is an antipodal and bipartite $(0,2)$-graph.

As noted in [2] and [7], a graph $G$ is a hypercube if and only if $G$ is spherical and bipartite. We aim to substitute this condition for a graph to be spherical with a weaker condition of antipodality and with a local condition for a graph to be a $(0,2)$-graph. Thus we have 
Corollary 5 A bipartite graph is spherical if and only if it is an antipodal $(0,2)$ graph.

Proof of Theorem 4. A hypercube is clearly an antipodal and bipartite $(0,2)$ graph. For the converse proof we assume that $G=(V, E)$ is an antipodal bipartite $(0,2)$-graph. Let $u$ and $v$ be adjacent vertices, whence $\left[u, v^{\prime}\right] \cup\left[v, u^{\prime}\right]=V$ and $\left[u, v^{\prime}\right] \cap\left[v, u^{\prime}\right]=\emptyset$.

We first prove the following result.

Claim. If $u, u_{1}, u_{2}, \ldots, u_{n}$ is a geodesic on $\left[u, v^{\prime}\right]$, then for any $u_{i}, i=1,2, \ldots, n$ there exists a unique vertex $v_{i} \in\left[v, u^{\prime}\right]$ such that $v_{i} \in N\left(u_{i}\right), v, v_{1}, v_{2}, \ldots, v_{n}$ is a geodesic on $\left[v, u^{\prime}\right]$ and $N\left(v_{i}\right) \cap\left[u, v^{\prime}\right]=\left\{u_{i}\right\}$.

Proof of Claim. We proceed by induction on $n$. If $n=1$, then $u_{1}$ is adjacent to $u$ and $d\left(u_{1}, v\right)=2$. Because $u \in\left[u_{1}, v\right]$ and $G$ is a $(0,2)$-graph, there exists a vertex $v_{1}$ such that $\left[u, v_{1}\right]=\left[v, u_{1}\right]$. Clearly the path $u, v, v_{1}$ is a geodesic. If $v_{1} \in\left[u, v^{\prime}\right]$, there exists a geodesic $u, v, v_{1}, \ldots, v^{\prime}$, which is a contradiction. Thus $v_{1} \in\left[v, u^{\prime}\right]$. Let $w$ be a vertex such that $w \in N\left(u_{1}\right) \cap\left[v, u^{\prime}\right], w \neq v_{1}$. If $w \in N\left(v_{1}\right)$, there is a triangle $u_{1}, v_{1}, w, u_{1}$, which is a contradiction since $G$ is bipartite. If $v, u, u_{1}, w$ is a geodesic, then $u \in\left[v, u^{\prime}\right]$, because $w \in\left[v, u^{\prime}\right]$; a contradiction. If $d(v, w)=2$, there exists a vertex $w_{1} \in\left[v, u^{\prime}\right]$ such that $w, w_{1}, v$ is a geodesic. This implies an odd cycle $v, u, u_{1}, w, w_{1}, v$, which is a contradiction. Thus $d(v, w)=1$ and then $w \in\left[v, u_{1}\right]$. This contradicts the fact that $G$ is a $(0,2)$-graph, $d\left(u, v_{1}\right)=d(u, w)=2$, and $u, v_{1}, w \in\left[v, u_{1}\right]$. Thus the Claim holds for $n=1$.

Assume now, that the Claim holds for all $n \leq k-1$. Let $u, u_{1}, u_{2}, \ldots, u_{k}$ be a geodesic on $\left[u, v^{\prime}\right]$. By the induction assumption there exists a geodesic $v, v_{1}, v_{2}, \ldots$, $v_{k-1}$ such that $v_{i} \in N\left(u_{i}\right) \cap\left[v, u^{\prime}\right]$ and $N\left(v_{i}\right) \cap\left[u, v^{\prime}\right]=\left\{u_{i}\right\}$ for all $i=1,2, \ldots, k-1$.

Because $d\left(u_{k}, v_{k-1}\right)=2, u_{k-1} \in\left[u_{k}, v_{k-1}\right]$, and because $G$ is a $(0,2)$-graph, there exists a vertex $v_{k} \neq u_{k-1}$ adjacent to $u_{k}$ and $v_{k-1}$. By induction assumption $\left\{u_{k-1}\right\}=$ $N\left(v_{k-1}\right) \cap\left[u, v^{\prime}\right]$, and thus we have $v_{k} \in\left[v, u^{\prime}\right]$. Because $d\left(v, v_{k-1}\right)=k-1$ and $v_{k}$ is adjacent to $v_{k-1}$, we have $k-2 \leq d\left(v, v_{k}\right) \leq k$. If $d\left(v, v_{k}\right)=k-2$, there exists a geodesic $u, v, v_{1}, \ldots, v_{k}, u_{k}$, whence $v \in\left[u, v^{\prime}\right]$, which is a contradiction. If $d\left(v, v_{k}\right)=k-1$, there exists a cycle $u, u_{1}, u_{2}, \ldots, u_{k}, v_{k}, v_{k-1}, \ldots, v, u$ such that the length of the cycle is $k+1+(k-1)+1=2 k+1$, a contradiction. Thus $d\left(v, v_{k}\right)=k$ and $v, v_{1}, \ldots, v_{k-1}, v_{k}$ is a geodesic.

If there exists a vertex $z \in N\left(u_{k}\right) \cap\left[v, u^{\prime}\right], z \neq v_{k}$, then $z \notin N\left(v_{k}\right)$, because otherwise $u_{k}, v_{k}, z, u_{k}$ is a triangle. Thus $d\left(v_{k}, z\right)=2$. Because $d\left(u_{k-1}, z\right)=2, u_{k} \in$ $\left[u_{k-1}, z\right]$, and $G$ is a $(0,2)$-graph, there exists a vertex $w$ such that $\left[u_{k}, w\right]=\left[u_{k-1}, z\right]$. Clearly $w \in N\left(u_{k-1}\right) \cap N(z)$. Two cases arise (i) $w \in\left[v, u^{\prime}\right]$ (ii) $w \in\left[u, v^{\prime}\right]$.

(i) If $w \in\left[v, u^{\prime}\right]$, then by induction assumption $w=v_{k-1}$, and $z, v_{k}, u_{k-1} \in$ $\left[u_{k}, v_{k-1}\right]$. Because $d\left(u_{k-1}, z\right)=d\left(u_{k-1}, v_{k}\right)=2$ and $G$ is a $(0,2)$-graph we have a 
contradiction.

(ii) Since $w \in N\left(u_{k-1}\right)$ and $d\left(u, u_{k-1}\right)=k-1$, the relation $w \in N\left(u_{k-1}\right)$ implies $k-2 \leq d(u, w) \leq k$. If $d(u, w)=k-2$, then $z \in N(w) \cap\left[v, u^{\prime}\right]$ and the induction assumption imply $d(v, z)=k-2$. Because $z, u_{k}, v_{k}$ is a geodesic and $d\left(v, v_{k}\right)=k$, the vertex $u_{k}$ is on the $v, v_{1}, \ldots, v_{k}$ geodesic. Now, by the relation $v_{k} \in\left[v, u^{\prime}\right]$ we have $u_{k} \in\left[v, u^{\prime}\right]$, which is a contradiction. Because $z \in N(w) \cap\left[v, u^{\prime}\right]$ and the induction assumption, the assumption $d(u, w)=k-1$ implies $d(v, z)=k-1$. But then there exists a cycle $u, u_{1}, \ldots, u_{k}, z, \ldots, v, u$ of length $k+1+k-1+1=$ $2 k+1$; a contradiction. Since $w$ is adjacent to $u_{k-1}$, the relation $d(u, w)=k$ implies that the path $u, u_{1}, \ldots, u_{k-1}, w$ is a geodesic. By the first part of the proof of the Claim, there exists a geodesic $v, z_{1}, z_{2}, \ldots, z_{k-1}, z$ on $\left[v, u^{\prime}\right]$ such that $z_{i} \in N\left(u_{i}\right)$ for all $i=1,2, \ldots k-1$. By induction assumption, $z_{k-1}=v_{k-1}$. This implies that $u_{k-1}, z, v_{k} \in\left[v_{k-1}, u_{k}\right]$ and $d\left(u_{k-1}, z\right)=d\left(u_{k-1}, v_{k}\right)=2$ which is a contradiction, because $G$ is a $(0,2)$-graph and $d\left(v_{k-1}, u_{k}\right)=2$. If we assume that there exists $z \in\left[u, v^{\prime}\right] \cap N\left(v_{k}\right), z \neq u_{k}$, then by symmetry this yields a contradiction. Thus the Claim holds for all $n$.

To prove Theorem 4 , we proceed by induction on $|V|$ of $G$. If $|V|=4$, then clearly $G$ is $Q_{2}$. Assume that the theorem holds for $|V| \leq k$, and let $u$ and $v$ be adjacent vertices. By the Claim, the subgraphs induced by $\left[u, v^{\prime}\right]$ and $\left[v, u^{\prime}\right]$ are isomorphic, and moreover, $G$ is isomorphic to the graph $Q_{1} \times G_{0}$ where $G_{0}$ is isomorphic to the subgraph induced by $\left[u, v^{\prime}\right]$. By the induction assumption, it suffices to prove that the subgraph $G_{1}$ of $G$ induced by $\left[u, v^{\prime}\right]$ is an antipodal and bipartite $(0,2)$-graph.

Because $G$ is bipartite, the subgraph $G_{1}$ is also bipartite. It follows from the Claim that every $x-y$ geodesic, $x, y \in\left[u, v^{\prime}\right]$, contains $p$ vertices of $\left[v, u^{\prime}\right]$, where $p$ is zero or an even number. Thus if $d(x, y)=2$ and $x, y \in\left[u, v^{\prime}\right]$, then $[x, y]$ does not contain any vertices of $\left[v, u^{\prime}\right]$. Thus $[x, y] \subseteq\left[u, v^{\prime}\right]$ and $G_{1}$ is a $(0,2)$-graph, since $G$ is a $(0,2)$-graph.

Assume that $u_{1} \in\left[u, v^{\prime}\right]$. By the Claim there is a unique vertex $v_{1} \in N\left(u_{1}\right) \cap\left[v, u^{\prime}\right]$ and thus $v_{1}^{\prime} \in\left[u, v^{\prime}\right]$. To prove the antipodality of $G_{1}$ it suffices to prove that $\left[u_{1}, v_{1}^{\prime}\right]=\left[u, v^{\prime}\right]$.

Assume first, that $z \in\left[u, v^{\prime}\right]$. Since $z \in\left[v_{1}, v_{1}^{\prime}\right]$, there exists a geodesic $v_{1}, z_{1}, \cdots$, $z_{n-1}, z, \cdots, v_{1}^{\prime}$. Since $z, u_{1} \in\left[u, v^{\prime}\right]$ and $v_{1}$ is adjacent to $u_{1}$, there exists, by the Claim, a geodesic $u_{1}, w_{1}, w_{2}, \cdots, w_{n-1}, z, \cdots, v_{1}^{\prime}$ such that $w_{i}=z_{i}$, if $z_{i} \in\left[u, v^{\prime}\right]$ and $w_{i} \in N\left(z_{i}\right) \cap\left[u, v^{\prime}\right]$, if $z_{i} \in\left[v, u^{\prime}\right]$. By the Claim, the vertex $w_{i}$ is unique for all $i=1, \ldots, n-1$, and thus $z \in\left[u_{1}, v_{1}^{\prime}\right]$.

To prove the another inclusion we assume that there exists a geodesic $u_{1}, \cdots, z_{1}$, $z_{2}, \cdots, z_{l}, \cdots, v_{1}^{\prime}$, such that $z_{1}, z_{l} \in\left[u, v^{\prime}\right]$ and $z_{2}, \ldots z_{l-1} \in\left[v, u^{\prime}\right]$. We may assume now that the $u_{1}-z_{1}$ geodesic does not contain any vertex of $\left[v^{\prime}, u\right]$. If $l=3$, then 
$z_{1}, z_{l} \in N\left(z_{2}\right) \cap\left[v, u^{\prime}\right]$, which contradicts the Claim. Thus $z_{3} \in\left[v, u^{\prime}\right]$. Because $G$ is a $(0,2)$-graph and $d\left(z_{1}, z_{3}\right)=2$, there exists a vertex $w_{2} \in N\left(z_{1}\right) \cap N\left(z_{3}\right)$, $w_{2} \neq z_{2}$. By the Claim, $N\left(z_{1}\right) \cap\left[v, u^{\prime}\right]=\left\{z_{2}\right\}$, and thus $w_{2} \in\left[u, v^{\prime}\right]$. By repeating the process above we conclude, that there exists a geodesic $z_{1}, w_{2}, w_{3}, \cdots, w_{l-1}$, such that $w_{i} \in N\left(z_{i}\right) \cap\left[u, v^{\prime}\right]$ for all $i=2,3, \ldots, l-1$. Since $z_{l}, w_{l-1} \in N\left(z_{l-1}\right) \cap\left[u, v^{\prime}\right]$, the Claim implies $w_{l-1}=z_{l}$, which contradicts the fact that the path $z_{1}, z_{2} \cdots, z_{l}$ goes along a $u_{1}-v_{1}^{\prime}$ geodesic. Thus any vertex on the $u_{1}-v_{1}^{\prime}$ geodesic is in the interval $\left[u, v^{\prime}\right]$. This completes the proof.

\section{Prime convex intersection characterization}

A nonempty vertex set $A \subset V$ is a convex, if $x, y \in A$ and $z$ on an $x-y$ geodesic imply that $z \in A$. Clearly a nonempty intersection $A \cap B$ of two convexes is a convex, too. By $\langle D>$ we denote the least convex containing the vertex set $D$ : $<D>=\bigcap\{C \mid C$ is a convex and $D \subset C\}$. The least convex containing the vertices $x$ and $y$ is briefly denoted by $\langle x, y\rangle$. In general, the convex $\langle x, y\rangle$ also contains vertices not on an $x-y$ geodesic and hence $[x, y] \subset<x, y>$. In the covering graph of a finite distributive lattice, $[x, y]=\langle x, y\rangle$ for each two vertices $x$ and $y$, and thus in each (finite) hypercube $\langle x, y>\subset[x, y]$ for all $x, y \in V$. A convex $P \neq V$ is called prime if also the set $V \backslash P=\bar{P}$ is a convex. A graph $G$ is a prime convex intersection graph if each convex $C$ of $G$ is the intersection of prime convexes containing $C$ : $C=\bigcap\{P \mid P$ is a prime convex and $C \subset P\}$. For example, all nontrivial trees and all nontrivial complete graphs are prime convex intersection graphs. As the definition shows, prime convexes exist in pairs and this property is used in our characterization

Theorem 6 A graph $G$ is an $n$-cube $Q_{n}$ if and only if

(i) there are $n$ disjoint pairs of prime convexes in $G$;

(ii) $G$ is a prime convex intersection graph;

(iii) each intersection of $n$ prime convexes, no one of which is from the same pair, is a vertex of $G$.

Proof. Let $G$ have the properties $(i)-($ iii $)$. By Theorem 4 proving that $G$ is a hypercube it suffices to prove that $G$ is an antipodal and bipartite $(0,2)$-graph. We prove this by sequence of claims given and proved below.

Claim 1. Each prime convex $P_{i}$ of $G$ is maximal, i.e. there is no prime convex $P_{j}$ in $G$ containing $P_{i}$ properly.

Proof of Claim 1. Assume that $P_{1} \subset P_{2}$ with $P_{1} \neq P_{2}$. By (iii), there exists a vertex $a$ of $G$ such that $\{a\}=P_{1} \cap P_{2} \cap P_{3} \cap \ldots \cap P_{n}$. Because $P_{1} \subset P_{2}$, we have 
$P_{1} \cap \bar{P}_{2}=\emptyset$ and thus $\emptyset=P_{1} \cap \bar{P}_{2} \cap P_{3} \cap \ldots \cap P_{n}$ contradicts the property (iii), and the Claim 1 follows.

If $a b$ is an edge of $G, a \in P_{i}$ and $b \in \bar{P}_{i}$, we say that the pair $P_{i}, \bar{P}_{i}$ of prime convexes cuts off the edge $a b$.

Claim 2. If a is a vertex of $G$, then each pair $P_{i}, \bar{P}_{i}, i=1, \ldots, n$ cuts off exactly one edge incident to a.

Proof of Claim 2. Because $G$ is a prime convex intersection graph and the vertex $a$ is obtained as an intersection of prime convexes, the pairs of prime convexes of $G$ must cut off each edge incident to $a$. If there is a pair of prime convexes, say $P_{1}, \bar{P}_{1}$, such that $a \in P_{1}$ but the pair does not cut off any edge incident to $a$, then the vertex $a$ has an intersection representation $\{a\}=\bigcap\left\{P_{i} \mid i=2,3, . ., n\right\}$, and because $a \in P_{1}, a$ also has the representation $\{a\}=\bigcap\left\{P_{i} \mid i=1,2, \ldots, n\right\}$. On the other hand, $a \notin \bar{P}_{1}$, and thus $\bar{P}_{1} \cap P_{2} \cap \ldots \cap P_{n}=\emptyset$, which contradicts (iii), and thus each pair of prime convexes cuts off at least one edge incident to $a$. Let now $a \in P_{1}$ and the pair $P_{1}, \bar{P}_{1}$ cut off at least two edges $a b_{1}$ and $a b_{2}$. If the edge $b_{1} b_{2}$ does not exist in $G$, then one of the $b_{1}-b_{2}$ geodesics goes through $a$, and thus $a \in \bar{P}_{1}$; a contradiction. Thus we assume that the edge $b_{1} b_{2}$ exists in $G$ and the vertices $a, b_{1}, b_{2}$ induce a complete subgraph $K_{3}$ of $G$. In order to obtain the vertex $b_{1}$ as an intersection of prime convexes, there must be a pair cutting off all edges incident to $b_{1}$ or to $b_{2}$ in $K_{3}$. Assume that the pair $P_{2}, \bar{P}_{2}$ cuts off the edges $a b_{1}$ and $b_{1} b_{2}$ and $b_{1} \in \bar{P}_{2}$, and thus the edge $a b_{1}$ is cutted off by at least two pairs $P_{1}, \bar{P}_{1}$ and $P_{2}, \bar{P}_{2}$. The relation $P_{2} \subset \bar{P}_{1}$ is a contradiction because each prime convex is maximal as stated above. Assume that $P_{2} \not \subset \bar{P}_{1}$, i.e. there is a vertex $c$ belonging to the intersection $P_{1} \cap P_{2}$. Because $G$ is a prime convex intersection graph and $\left\{a, b_{1}\right\}$ is a convex as a set of end vertices of an edge, we have $\left\{a, b_{1}\right\}=\bigcap\left\{P_{i} \mid a, b_{1} \in P_{i}\right\}$. Thus $\{a\}=\bar{P}_{2} \cap\left(\bigcap\left\{P_{i} \mid a, b_{1} \in P_{i}\right\}\right)=P_{1} \cap \bar{P}_{2} \cap\left(\bigcap\left\{P_{i} \mid a, b_{1} \in P_{i}\right\}\right)$ containing by (iii) at most $n$ prime convexes from $n$ disjoint pairs of prime convexes. Now $P_{1} \cap P_{2} \cap\left(\bigcap\left\{P_{i} \mid a, b_{1} \in P_{i}\right\}\right)=\emptyset$ because the vertex $c \in P_{1} \cap P_{2}$ cannot belong to the intersection $\left\{a, b_{1}\right\}=\bigcap\left\{P_{i} \mid a, b_{1} \in P_{i}\right\}$. This contradicts (iii), and the Claim 2 holds.

Claim 2 also implies that each vertex of $G$ has degree $n$ i.e. $G$ is regular of degree $n$.

Claim 3. The graph $G$ is bipartite.

Proof of Claim 3. If a prime convex $P_{i}$ cuts off an edge of a cycle it must cut off also another edge of the cycle (i.e. cut off the cycle), because $P_{i}$ induces a connected subgraph of $G$. If there is an odd cycle in $G$ there also is a least odd cycle $C$ without chords in $G$. If $P_{i}$ cuts off an edge of $C$, it also cuts off an opposite edge because otherwise $P_{i}$ or $\bar{P}_{i}$ is not a convex. Each edge of $C$ must be cutted off by prime 
convexes; if there is an uncutted edge $a b$ then its endpoints do not have a prime convex intersection representation which contradicts $(i i)$. Because $C$ is odd, one edge must be cutted off twice, which contradicts the proof of the Claim 2 and Claim 3 holds.

Claim 4. The graph $G$ is antipodal.

Proof of Claim 4. If $\{a\}=P_{1} \cap P_{2} \cap \ldots \cap P_{n}$ we denote the set $\bar{P}_{1} \cap \bar{P}_{2} \cap \ldots \cap \bar{P}_{n}$ by $\left\{a^{\prime}\right\}$ and call $a^{\prime}$ the complement of $a$. Let $d\left(a, a^{\prime}\right)=l$. By the definitions of $a$ and $a^{\prime}$, no prime convex of $G$ can contain simultaneously the vertices $a$ and $a^{\prime}$. Moreover, any prime convex $P_{k}$ cannot simultaneously cut off (at least) two edges of an $x-x^{\prime}$ geodesic: if $P_{k}$ cuts off the edges $e_{1} e_{2}$ and $e_{r} e_{s}$ of an $x-x^{\prime}$ geodesic such that $e_{2}, e_{r} \in P_{k}$ and $e_{1}, e_{s} \in \bar{P}_{k}$, then $e_{2}, e_{r} \in \bar{P}_{k}$, because $e_{2}$ and $e_{r}$ are on the $e_{1}-e_{s}$ geodesic. In order to obtain each vertex of an $x-x^{\prime}$ geodesic as prime convex intersection, each edge of that $x-x^{\prime}$ geodesic must be cutted off exactly once. If $l<n$, there is a pair $P_{j}, \bar{P}_{j}$ not cutting of any edge of $x-x^{\prime}$ geodesic, and thus $x, x^{\prime} \in P_{j}$ or $x, x^{\prime} \in \bar{P}_{j}$, which is a contradiction. Because each edge of $x-x^{\prime}$ geodesic must be cutted off, the relation $l>n$ cannot hold, and thus we have $l=n$. By the definition of $a^{\prime}$, there is for any vertex $a$ exactly one vertex $a^{\prime}$. If $x \neq y$ for two vertices $x$ and $y$ of $G$, there must be at least one pair, say $P_{t}, \bar{P}_{t}$, such that $y \in P_{t}$ and $x \notin P_{t}$. This means that $y \notin \bar{P}_{t}$ and $x \in \bar{P}_{t}$, whence $y^{\prime} \in \bar{P}_{t}, x^{\prime} \in P_{t}$, and $x^{\prime} \neq y^{\prime}$. Hence for any vertex $a$ of $G$ there is a unique vertex $a^{\prime}$ such that $d\left(a, a^{\prime}\right)=n$.

The vertices in $P_{1}$ induce a connected subgraph $G_{n-1}^{1}$ of the graph $G$ which we shall here denote by $G_{n}\left(G=G_{n}\right)$. If $P_{i}$ is a prime convex of $G_{n}$, there are $n-1$ pairs of prime convexes $P_{1} \cap P_{i}, P_{1} \cap \bar{P}_{i}(i=2,3, \ldots, n)$ in $G_{n-1}^{1}$. This proves ( $i)$ for $G_{n-1}^{1}$. If $a$ is vertex in $P_{1}$, then we have by $($ iii $)$ of $G_{n}$ the representation $\{a\}=P_{1} \cap P_{2} \cap P_{3} \cap \ldots \cap P_{n}=\left(P_{1} \cap P_{2}\right) \cap\left(P_{1} \cap P_{3}\right) \cap \ldots \cap\left(P_{1} \cap P_{n}\right)$, which is a representation of $a$ as an intersection of $n-1$ prime convexes of $G_{n-1}^{1}$. Thus each prime convex intersection representation of a convex in $G_{n-1}^{1}$ can be obtained from the corresponding representation in $G_{n}$. This shows that $G_{n-1}^{1}$ has the properties (ii) and $($ iii $)$. The result holds also if $P_{1}$ is replaced by any of the prime convexes $P_{i}, \bar{P}_{i}(i=2,3, \ldots, n)$ and $\bar{P}_{1}$. Because $G_{n-1}^{1}$ has the same properties as $G=G_{n}$, we can deduce from $G_{n-1}^{1}$ (and from all other graphs deduced from $G_{n}$ by the same way as $\left.G_{n-1}^{1}\right)$ graph $G_{n-2}^{12}$. The graph $G_{n-2}^{12}$ is induced by the vertices in the set $P_{1} \cap P_{2}$ and there are $n-2$ pairs $\left(P_{1} \cap P_{2}\right) \cap P_{i},\left(P_{1} \cap P_{2}\right) \cap \bar{P}_{i}(i=3,4, \ldots, n)$ of prime convexes in $G_{n-2}^{12}$. As above, we see, that $G_{n-2}^{12}$ has the properties $(i)-($ iii $)$. By continuing this process, the vertex set $P_{1} \cap P_{2} \cap \ldots \cap P_{n-2}$ induces the graph $G_{2}^{12 \ldots n-2}$ having two pairs $\left(P_{1} \cap \ldots \cap P_{n-2}\right) \cap P_{j},\left(P_{1} \cap \ldots \cap P_{n-2}\right) \cap \bar{P}_{j}(j=n-1, n)$ of prime convexes and having the properties $(i)-(i i i)$ of the theorem. Thus the properties proved to hold for $G=G_{n}$ also hold for $G_{2}^{12 \ldots n-2}$, and accordingly, $G_{2}^{12 \ldots n-2}$ is regular of 
degree 2 and there are four vertices obtained as intersections of the prime convexes: $\left\{c_{1}\right\}=\left(P_{1} \cap \ldots \cap P_{n-2}\right) \cap P_{n-1} \cap\left(P_{1} \cap \ldots \cap P_{n-2}\right) \cap P_{n},\left\{c_{2}\right\}=\left(P_{1} \cap \ldots \cap P_{n-2}\right) \cap$ $P_{n-1} \cap\left(P_{1} \cap \ldots \cap P_{n-2}\right) \cap \bar{P}_{n},\left\{c_{3}\right\}=\left(P_{1} \cap \ldots \cap P_{n-2}\right) \cap \bar{P}_{n-1} \cap\left(P_{1} \cap \ldots \cap P_{n-2}\right) \cap P_{n}$ and $\left\{c_{4}\right\}=\left(P_{1} \cap \ldots \cap P_{n-2}\right) \cap \bar{P}_{n-1} \cap\left(P_{1} \cap \ldots \cap P_{n-2}\right) \cap \bar{P}_{n}$. By the definition, $c_{4}=c_{1}^{\prime}$ and $c_{3}=c_{2}^{\prime}$. Now the proof of the properties of $G_{n}$ implies that $d\left(c_{1}, c_{1}^{\prime}\right)=$ $2=d\left(c_{2}, c_{2}^{\prime}\right)$ in $G_{2}^{12 \ldots n-2}$. These properties imply that $G_{2}^{12 \ldots n-2}$ is a four-cycle, where $\left[x, x^{\prime}\right]=V\left(G_{2}^{12 \ldots n-2}\right)$ for each vertex $x$ of $G_{2}^{12 \ldots n-2}$. By using induction, we assume that $\left[x, x^{\prime}\right]=V\left(G_{n-j}^{12 \ldots j}\right), j=1,2, \ldots, n-2$ and thus $\left[x, x^{\prime}\right]=V\left(G_{n-1}^{1}\right)$ for each vertex $x$ of $G_{n-1}^{1}$, and prove that $\left[x, x^{\prime}\right]=V\left(G_{n}\right)$ for each vertex $x$ of $G_{n}$. Let the vertex $x$ have the representation $\{x\}=P_{1} \cap P_{2} \cap P_{3} \cap \ldots \cap P_{n}$ in $G_{n}$ and thus the same vertex has in the graph $G_{n-1}^{1}$ induced by the vertex set $P_{1}$ the representation $\{x\}=\left(P_{1} \cap P_{2}\right) \cap\left(P_{1} \cap\right.$ $\left.P_{3}\right) \cap \ldots \cap\left(P_{1} \cap P_{n}\right)=P_{1} \cap\left(P_{2} \cap P_{3} \cap \ldots \cap P_{n}\right)$. The complement $c$ of $x$ in the graph $G_{n-1}^{1}$ is by the definition $\left(P_{1} \cap \bar{P}_{2}\right) \cap\left(P_{1} \cap \bar{P}_{3}\right) \cap \ldots \cap\left(P_{1} \cap \bar{P}_{n}\right)=P_{1} \cap\left(\bar{P}_{2} \cap \bar{P}_{3} \cap \ldots \cap \bar{P}_{n}\right)=\{c\}$. By the induction assumption, $[x, c]=V\left(G_{n-1}^{1}\right)$ and by the proof above $d(x, c)=n-1$ in $G_{n-1}^{1}$ as well as in $G_{n}$. The representations $\left\{x^{\prime}\right\}=\bar{P}_{1} \cap \bar{P}_{2} \cap \bar{P}_{3} \cap \ldots \cap \bar{P}_{n}$ and $\{c\}=P_{1} \cap\left(\bar{P}_{2} \cap \bar{P}_{3} \cap \ldots \bar{\cap} P_{n}\right)$ show that $x^{\prime}, c \in \bar{P}_{2} \cap \bar{P}_{3} \cap \ldots \cap \bar{P}_{n}$. Because a pair of prime convexes cuts off exactly one edge incident to a vertex of $G_{n}$, the prime convex $P_{1}$ (as well as $\bar{P}_{1}$ ) cuts off the edge $x^{\prime} c$ and $\bar{P}_{2} \cap \bar{P}_{3} \cap \ldots \cap \bar{P}_{n}=\left\{x^{\prime}, c\right\}$. Because $d\left(x, x^{\prime}\right)=n=d(x, c)+1$ in $G_{n}$, we see that each vertex of $[x, c]=V\left(G_{n-1}^{1}\right)=P_{1}$ is on an $x-x^{\prime}$ geodesic in $G_{n}$. By repeating the proof for the subgraph $G_{n-1}^{\overline{1}}$ induced by $\bar{P}_{1}$ in $G_{n}$ we see that each vertex in $V\left(G_{n-1}^{\overline{1}}\right)=\bar{P}_{1}$ is on an $x-x^{\prime}$ geodesic in $G_{n}$. Accordingly, $\left[x, x^{\prime}\right]=P_{1} \cup \bar{P}_{1}=V\left(G_{n}\right)$ for each vertex $x$ and its complement in $G_{n}$ and thus the graph $G=G_{n}$ is antipodal such that the complement $x^{\prime}$ is also the antipode of $x$ and Claim 4 holds.

Claim 5. $G$ is a $(0,2)$-graph.

Proof of Claim 5. Let $x$ and $y$ be two vertices of $G$ such that $d(x, y)=2$. Because $G$ is a prime convex intersection graph, $\langle x, y\rangle=\bigcap\left\{P_{i} \mid x, y \in P_{i}\right\}$. As seen above, each nonempty intersection of prime convexes induces a subgraph $G_{x y}$ of $G$ having the properties $(i)-(i i i)$. The vertex $x$ has a complement/an antipode $x^{\prime}$ in $G_{x y}$ such that $\left[x, x^{\prime}\right]=V\left(G_{x y}\right)$ and thus the vertex $y$ is on an $x-x^{\prime}$ geodesic in $G_{x y}$ as well as in $G$. If $y \neq x^{\prime}$, then $d\left(x, x^{\prime}\right)>d(x, y)=2$, and we can cut off the vertex $x^{\prime}$ from $G_{x y}$ by using prime convexes containing $x^{\prime}$. As a result we obtain a smaller convex containing $x$ and $y$, which is a contradiction. Thus $x^{\prime}=y, d\left(x, x^{\prime}\right)=2$, and $G_{x y}$ is a 4-cycle (without chords) of $G$. In a 4-cycle there exists for a vertex $z$ a unique vertex $z_{1}$ with $\left[z, z_{1}\right]$ containg all vertices of the 4-cycle. Hence the Claim 5 holds and so the conditions $(i)-($ iii) imply an $n$-hypercube by Theorem 4 .

Conversely, let $G$ be an $n$-hypercube $Q_{n}$. As well known, $Q_{n}=Q_{1} \times Q_{n-1}$, which means that $Q_{n}$ is obtained by combining two complementary $n-1$-hypercubes $Q_{n-1}^{1}$ 
and $Q_{n-1}^{2}$ such that if $f$ is an isomorphism between these two $n-1$-hypercubes then each vertex $x$ of $Q_{n-1}^{1}$ is joined by an edge to its image vertex $f(x)$ in $Q_{n-1}^{2}$. As known, an $n$-hypercube is a prime convex intersection graph where the vertex sets $V\left(Q_{n-1}^{1}\right), V\left(Q_{n-1}^{2}\right)$ of each pair of complementary $n-1$-hypercubes $Q_{n-1}^{1}$ and $Q_{n-1}^{2}$ constitute a pair of prime convexes. As well known, there are $n$ disjoint pairs of complementary $n-1$-hypercubes in an $n$-hypercube. Thus $(i)$ and $(i i)$ hold in the graph of $Q_{n}$. It is also known that the intersection of two $n-1$-hypercubes of an $n$-hypercube is an $n$-2-hypercube or an empty graph (when the $n$-1-hypercubes are the pair of complementary $n-1$-hypercubes of $Q_{n}$ ). We prove the validity of (iii) of $Q_{n}$ by induction on the dimension of the hypercube. One can see by inspection that ( iii) holds for $Q_{2}$ and we assume that (iii) holds for all $n-1$-hypercubes. Let $V\left(Q_{n-1}(j)\right) j=1, \ldots, n$ be $n$ prime convexes of $Q_{n}$, no one of which is from the same pair and consider the intersection $\bigcap\left\{V\left(Q_{n-1}(j)\right) \mid j=1, \ldots, n\right\}$. We can write this intersection as follows: $\bigcap\left\{V\left(Q_{n-1}(j)\right) \mid j=1, \ldots, n\right\}=\left(V\left(Q_{n-1}(1)\right) \cap V\left(Q_{n-1}(n)\right)\right) \cap$ $\left(V\left(Q_{n-1}(2)\right) \cap V\left(Q_{n-1}(n)\right)\right) \cap\left(V\left(Q_{n-1}(3)\right) \cap V\left(Q_{n-1}(n)\right)\right) \cap \ldots \cap\left(V\left(Q_{n-1}(n-1)\right) \cap\right.$ $\left.V\left(Q_{n-1}(n)\right)\right)$, where each set $V\left(Q_{n-1}(j)\right) \cap V\left(Q_{n-1}(n)\right)(j=1, \ldots, n-1)$ is the vertex set of an $n-2$-hypercube/prime convex in the $n-1$-hypercube induced by $V\left(Q_{n-1}(n)\right)$ in $Q_{n}$. By the assumption, this intersection in the $n-1$-hypercube is a vertex of $Q_{n-1}(n)$ which is contained in $Q_{n}$. This proves the assertion for $Q_{n}$ and

the characterization follows.

\section{References}

[1] A. Berman and A. Kotzig, Cross-Cloning and antipodal graphs, Discrete Mathematics 69 (1988), 107-114.

[2] A. Berrachedi, I. Havel and H.M. Mulder Spherical and clockwise spherical graphs, Czechoslovak Mathematical Journal 53 (2003), 295-309.

[3] A. Berrachedi and M. Mollard Median graphs and hypercubes, some new characterizations, Discrete Mathematics 208-209 (1999), 71-75.

[4] A. Berrachedi and M. Mollard On two problems about (0,2)-graphs and intervalregular graphs, Ars Combinatorics 49 (1998), 303-309.

[5] F. Göbel and H.J. Veldman Even graphs, Graph Theory 10 (1986), 225-239.

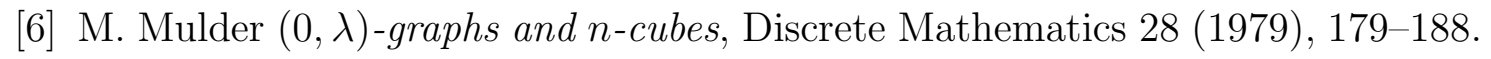

[7] W. Wenzel A sufficient condition for a bipartite graph to be a cube, Discrete Mathematics 259 (2002), 383-386. 